\title{
GMR
}

\section{Effect of overexpression of PTEN on apoptosis of liver cancer cells}

\author{
M.F. Li1 ${ }^{1 *}$, H. Guan ${ }^{2 *}$ and D.D. Zhang ${ }^{2,3}$ \\ ${ }^{1}$ Department of Hepatobiliary Surgery, Sichuan Academy of Medical Science, \\ Sichuan Provincial People's Hospital, Sichuan, China \\ ${ }^{2}$ Department of Health Management, Sichuan Academy of Medical Science, \\ Sichuan Provincial People's Hospital, Sichuan, China \\ ${ }^{3}$ Sichuan Key Laboratory for Disease Gene Study, \\ Sichuan Academy of Medical Science, Sichuan Provincial People's Hospital, \\ Sichuan, China \\ *These authors contributed equally to this study. \\ Corresponding author: D.D. Zhang \\ E-mail: zhangdd25@126.com
}

Genet. Mol. Res. 15 (2): gmr. 15028120

Received November 24, 2015

Accepted January 15, 2016

Published May 6, 2016

DOI http://dx.doi.org/10.4238/gmr. 15028120

\begin{abstract}
Liver cancer is a common malignant tumor associated with a short-survival period and high-mortality rate, and its prevalence in China is particularly high. This study aimed to investigate the effect of overexpressing the phosphatase and tensin homolog deleted on chromosome 10 (PTEN) gene on liver cancer cell apoptosis and provide new insight into the treatment of this disease. The experimental design included four treatment groups, consisting of HHCC and $\mathrm{H} 22$ cells transfected with PTEN recombinant plasmids (HHCC+PTEN, $\mathrm{H} 22+\mathrm{PTEN})$, and those transfected with control plasmids (HHCC+NC, $\mathrm{H} 22+\mathrm{NC})$. The expression of PTEN mRNA was determined by quantitative polymerase chain reaction, and protein levels were examined by western blot. Cell apoptosis was measured using flow cytometry and terminal deoxynucleotidyl transferase-mediated deoxyuridine triphosphate nick-end labeling. PTEN mRNA expression
\end{abstract}


in cells transfected with pcDNA3.1-PTEN was significantly increased compared to the control groups $(\mathrm{P}<0.05)$. In addition, western blotting revealed PTEN protein expression in the treatment groups to be significantly elevated in comparison to control cells $(\mathrm{P}<0.05)$. Flow cytometry showed that apoptosis rates of both HHCC + PTEN (approximately $21.9 \%$ ) and H22+PTEN (approximately $41.0 \%$ ) cells were significantly higher than those of the control groups $(\mathrm{P}<0.05)$. Moreover, the difference in apoptosis rate between experimental and control groups was significant $(\mathrm{P}<0.05)$. In this study, HHCC and $\mathrm{H} 22$ cells were successfully transfected with pcDNA3.1-PTEN in vitro. We conclude that overexpression of PTEN can effectively inhibit proliferation of these cells and promote their apoptosis.

Key words: PTEN; Transfection; Liver cancer; Apoptosis

\section{INTRODUCTION}

Liver cancer is a fatal malignant disease and constitutes the third leading cause of tumor mortality worldwide (Thomas et al., 2010). The prevalence of this disease is high in China, with both the number of new cases and deaths accounting for $54 \%$ of the global total (Wang et al., 2015). Liver cancer has become a major health concern in China. Currently, surgery remains the conventional approach to treatment. Owing to its high invasiveness, most cases of this disease have already progressed to intermediate and advanced stages by the time of diagnosis, meaning that the optimal time for surgery is missed. The resection rate for liver cancer is lower than $15 \%$, while the postoperative recurrence rate is approximately $50 \%$ (Siegel et al., 2015).

With the rapid development of molecular biological techniques, great advances have been made in our understanding of liver cancer at the molecular level. Moreover, the study of cancer cell proliferation and migration is of great significance to the diagnosis, treatment, and prognosis of related malignancies (Gupta and Massagué, 2006). Phosphatase and tensin homolog deleted on chromosome $10(P T E N)$ was one of the first observed tumor suppressor genes with phosphatase activity. Located on chromosome 10q23 and $200 \mathrm{~kb}$ in length, PTEN is a member of the protein tyrosine phosphatase gene family. It plays an important role in physiological activities involving cell apoptosis, adhesion, and migration (Shearn and Petersen, 2015). The frequency of loss of heterozygosity of PTEN in hepatocellular carcinoma is reported to be around 33\% (Yamazaki et al., 2015). Deletion and mutation of this gene may occur at high frequency during the progressive stage of metastatic malignancies affecting the brain (Carletti et al., 2015), endometrium (Clements et al., 2015), prostate (Manda et al., 2015), breast (Chen et al., 2016), lung (Dragoj et al., 2015), bone (Xi and Chen, 2015), colorectum (Atreya et al., 2013), and liver (Sendor et al., 2015). The loss of PTEN expression has been shown to be associated with aggressive tumor behavior. Therefore, we investigated the effect of overexpression of this gene on liver cancer cell apoptosis.

In this study, a eukaryotic expression vector carrying PTEN was transfected into human hepatic carcinoma cells (HHCC) and mouse hepatoma H22 cells to explore the effect of its overexpression on apoptosis, and further investigate its role in the development of liver cancer, to provide a primary scientific basis for the prevention and treatment of this disease. 


\section{MATERIAL AND METHODS}

\section{Instruments}

Polymerase chain reaction (PCR) thermal cycler (ABI 9700; Applied Biosystems, Foster City, CA, USA), real-time PCR instrument (Mx3000P; Stratagene, La Jolla, CA, USA), gel scanner (DF-23B; UVP, Cambridge, UK), flow cytometer (FACSCalibur; BD Biosciences, Franklin Lakes, NJ, USA), fluorescence microscope (LSM 510; Carl Zeiss, Oberkochen, Germany).

\section{Reagents}

TRIzol RNA extraction reagent (Invitrogen, Waltham, MA, USA), RNasin (Promega, Madison, WI, USA), fetal calf serum (Gibco, Waltham, MA, USA), annexin V/propidium iodide (PI) apoptosis kit (MultiSciences Biotech, Hangzhou, China), SYBR Green PCR Master Mix (purchased from Applied Biosystems), Lipofectamine transfection reagent (Invitrogen), antifade mounting medium and 4',6-diamidino-2-phenylindole (DAPI)-staining solution (Sigma-Aldrich, St. Louis, MO, USA), DL2000DNA marker (Dalian Proteri Biotechnology Co. Ltd., China), terminal deoxynucleotidyl transferase-mediated deoxyuridine triphosphate nick-end labeling (TUNEL) reagent kit (Roche, Basel, Switzerland).

\section{PTEN vector construction}

Genomic DNA was prepared from peripheral leukocytes. The primers (synthesized by Sangon Biotech, Shanghai, China) used to amplify PTEN were as follows: F, 5'-CCG GAA TTC ATG GCC ATG GCA ACC AAA GG-3'; and R, 5'-CCC AAG CTT TCA GAC TTT TGT AAT TTG TGT ATG C-3'. The reaction system was provided by Takara Bio Inc., and the product was run on a $1 \%$ agarose gel, from which it was extracted using a Gel Extraction Kit (Omega Bio-Tek Inc., Norcross, GA, USA). The product was then cloned into the pcDNA3.1 vector using HindIII and EcoRI restriction enzymes.

\section{Cell culture and transfection}

HHCC and H22 cells were purchased from ATCC (Manassas, VA, USA) and cultured in Dulbecco's modified Eagle's medium containing $10 \%$ fetal calf serum at $37^{\circ} \mathrm{C}$ in a $5 \% \mathrm{CO}_{2}$ atmosphere at saturated humidity. During the logarithmic phase, Lipofectamine 2000 was used to transfect empty pcDNA3.1 vectors and a pcDNA3.1-PTEN construct. The experiment comprised four treatment groups: pcDNA3.1 empty vector $+\mathrm{H} 22$ cells $(\mathrm{H} 22+\mathrm{NC})$, pcDNA3.1PTEN vector $+\mathrm{H} 22$ cells $(\mathrm{H} 22+\mathrm{PTEN})$, pcDNA3.1 empty vector $+\mathrm{HHCC}$ cells $(\mathrm{HHCC}+\mathrm{NC})$, and pcDNA3.1-PTEN vector + HHCC cells (HHCC+PTEN).

\section{Quantitative PCR (qPCR)}

Forty-eight hours after transfection, cells from each group were transferred into $1 \mathrm{~mL}$ TRIzol for total RNA extraction. Reverse transcription was then used to generate complementary DNA, $1 \mu \mathrm{L}$ of which was used to measure PTEN mRNA expression. The SYBR Green method was applied to perform qPCR, in which each sample was tested in triplicate. The primers used for $\mathrm{qPCR}$ are shown in Table 1. 
Table 1. Primers used for PTEN quantitative polymerase chain reaction.

\begin{tabular}{l|l}
\hline PTEN F & 5'-TAG ACC AGT GGC ACT GTT GT-3' \\
\hline PTEN R & 5'-TGG CAG ACC ACA AAC TGA GGA T-3' \\
\hline GAPDH F & 5'-CAG CCT CAA GAT CAT CAG CA-3' \\
\hline GAPDH R & 5'-TGT GGT CAT GAG TCC TTC CA-3' \\
\hline
\end{tabular}

$\mathrm{F}=$ forward; $\mathrm{R}=$ reverse.

\section{Western blot}

Forty-eight hours following transfection, cells from each group were collected by centrifugation. Radioimmunoprecipitation assay lysis buffer $(100 \mu \mathrm{L})$ was then added to lyse the cells on ice for $20 \mathrm{~min}$. Total protein concentration was measured by bicinchoninic acid assay prior to loading samples to ensure that all wells contained the same quantity of protein $(30 \mathrm{mg})$. Proteins were separated on a $10 \%$ separation gel before being wet-transferred to a polyvinylidene difluoride (PVDF) membrane for $30 \mathrm{~min}$ at $300 \mathrm{~mA}$. The primary antibody (anti-PTEN) was diluted 1:4000 in phosphate-buffered saline (PBS), and the PVDF membrane was then incubated with this antibody for $1 \mathrm{~h}$ at ambient temperature. Following this, Trisbuffered saline with Tween 20 (TBST) was used to wash the membrane three times $(5 \mathrm{~min} /$ wash). The secondary antibody was then added and incubated with the membrane for $1 \mathrm{~h}$ at ambient temperature before three further TBST washes.

\section{Cells apoptosis detection}

Forty-eight hours after plasmid transfection, cells were treated with $0.5 \%$ pancreatin and washed with $1 \mathrm{X}$ PBS. Cells were then resuspended in pre-cooled $1 \mathrm{X}$ binding buffer solution for a concentration of $1 \times 10^{6}$ cells $/ \mathrm{mL}$. Fluorescein isothiocyanate (FITC)-conjugated annexin $\mathrm{V}(1.25 \mu \mathrm{L})$ was added and reacted with cells in a light-free environment for $15 \mathrm{~min}$. The cells were then centrifuged for $5 \mathrm{~min}$ at ambient temperature and resuspended in $0.5 \mathrm{~mL}$ pre-cooled $1 \mathrm{X}$ binding buffer solution. PI $(10 \mu \mathrm{L})$ was then added and cells were kept on ice in a dark place before being analyzed by flow cytometry.

\section{TUNEL assay}

The culture medium was discarded and cells were transferred to a plate and washed three times with PBS before being fixed with 4\% paraformaldehyde for $20 \mathrm{~min}$. After three further PBS washes, the fixed cells were incubated with $0.3 \%$ PBS-Triton X-100 for $20 \mathrm{~min}$ and washed again three times with PBS. A mixture of TUNEL enzyme solution and TUNEL label solution (1:9) was then prepared, $50 \mu \mathrm{L}$ of which was added to each well and incubated with cells at $37^{\circ} \mathrm{C}$ for $1 \mathrm{~h}$, prior to being washed three times with PBS. DAPI-staining solution was added and incubated for 10 min at ambient temperature, and cells were then analyzed under a fluorescence microscope.

\section{Statistical analysis}

Statistical significance was defined as $\mathrm{P}<0.05$, and data were analyzed by SPSS version 10.0 (SPSS Inc., Chicago, IL, USA). The $t$-test was used to make comparisons between the two groups, while multi-group data were analyzed with a multi-factor $Q$-test. 


\section{RESULTS}

\section{PTEN vector construction}

PCR products were examined by $1 \%$ agarose gel electrophoresis (Figure 1), which showed an objective band of $1212 \mathrm{bp}$. The subsequently constructed plasmid was sent to Life Technologies Corporation (Shanghai, China) for sequencing, the results of which were verified using the Basic Local Alignment Search Tool and GenBank.

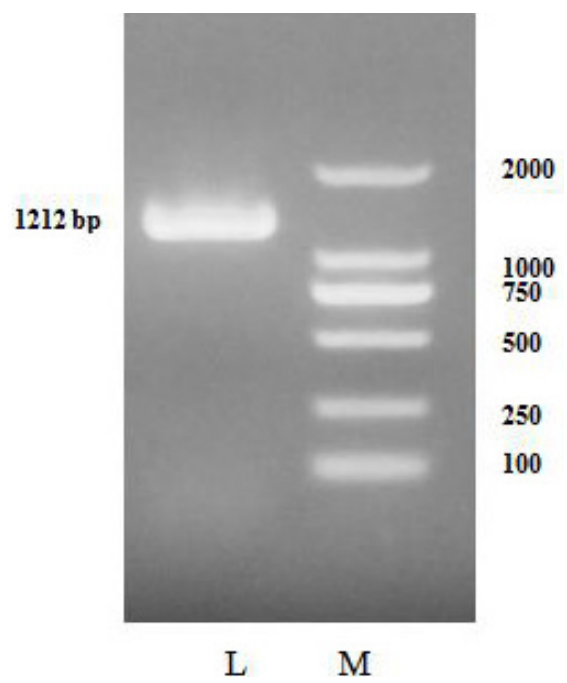

Figure 1. Agarose gel electrophoretogram of the PTEN polymerase chain reaction (PCR) product. Lane $M=$ DL2000 DNA marker; lane $L=$ PCR product.

\section{PTEN expression in $\mathrm{H} 22$ and HHCC cells measured by qPCR}

Forty-eight hours after transfection, PTEN expression in each group was determined by qPCR (Figure 2). Compared to the HHCC+NC group, relative PTEN expression in the HHCC+PTEN group was increased more than 9-fold $(9.26 \pm 0.4674)$. Moreover, this result was statistically significant $(t=17.02, \mathrm{P}=0.0034)$. Relative PTEN levels in $\mathrm{H} 22+\mathrm{PTEN}$ cells were more than 1800 times higher $(1877.93 \pm 191.043)$ than those of $\mathrm{H} 22+\mathrm{NC}$ cells, and this difference was also found to be significant $(t=30.63, \mathrm{P}=0.0011)$.

\section{PTEN expression in $\mathrm{H} 22$ and $\mathrm{HHCC}$ cells measured by western blot}

Forty-eight hours after transfection, the expression of PTEN protein in each group was examined by western blot (Figure 3 ). In the HHCC + NC and HHCC+PTEN groups, values were relative to GAPDH expression, PTEN expression was found to be $0.072 \pm 0.0096$ and $0.134 \pm 0.0094$, respectively. Statistical analysis revealed this to be a significant difference $(t$ $=8.14, \mathrm{P}=0.0012)$. Meanwhile, $\mathrm{H} 22+\mathrm{NC}$ and $\mathrm{H} 22+\mathrm{PTEN}$ cells demonstrated relative PTEN levels of $0.056 \pm 0.0086$ and $0.149 \pm 0.0077$, respectively. This difference between treatment and control groups was also found to be statistically significant $(t=14.04, \mathrm{P}=0.0001)$. 


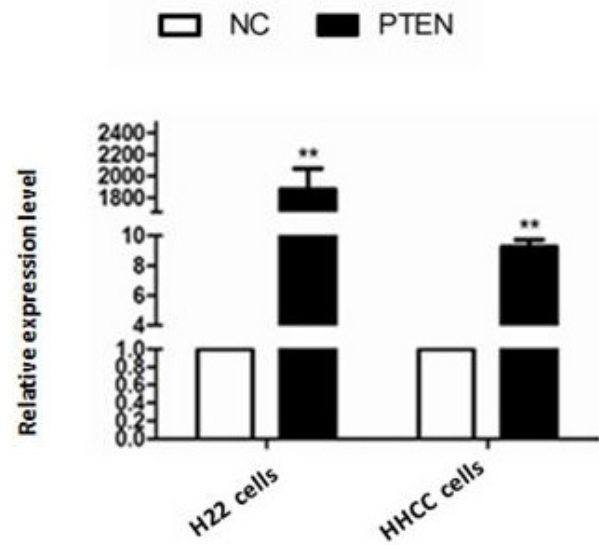

Figure 2. PTEN expression level in each group, measured by quantitative polymerase chain reaction. $\mathrm{NC}=$ transfected with empty vector, $\mathrm{PTEN}=$ transfected with $P T E N$ vector. $* * \mathrm{P}<0.01$.

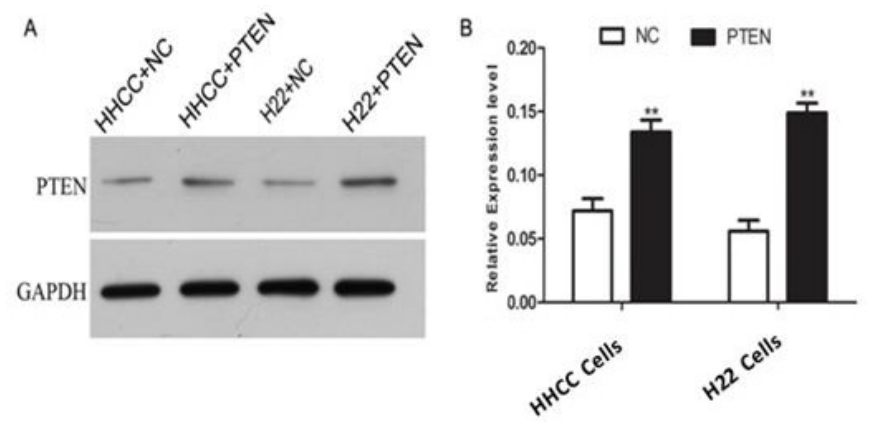

Figure 3. PTEN protein expression in each group, measured by western blotting. A. Western blot showing PTEN expression in each group. B. Quantified PTEN western blot, using gray values of each band; $\mathrm{NC}=$ transfected with empty vector, $\mathrm{PTEN}=$ transfected with $P T E N$ vector.

\section{Effect of PTEN overexpression on $\mathrm{H} 22$ and $\mathrm{HHCC}$ cell apoptosis}

Forty-eight hours after transfection, cells from each group were stained with FITCconjugated annexin $\mathrm{V}$, and apoptosis was detected by flow cytometry (Figure 4). The apoptosis rate of cells in the H22+PTEN group was revealed to be $41.0 \%$, significantly higher than that of the $\mathrm{H} 22+\mathrm{NC}$ group (chi-square $=34.07, \mathrm{P}=0.000$ ). Apoptosis of HHCC $+\mathrm{PTEN}$ cells was measured as $21.9 \%$, a rate significantly higher than that of cells in the HHCC+NC control group (chi-square $=12.37, \mathrm{P}=0.001)$.

\section{Effect of PTEN overexpression on $\mathrm{H} 22$ and HHCC apoptosis measured by TUNEL assay}

Forty-eight hours following transfection, the effect of PTEN overexpression on $\mathrm{H} 22$ and HHCC cell apoptosis was detected using a TUNEL assay (Figure 5). Compared to their corresponding controls, apoptosis rates in $\mathrm{H} 22(t=45.12, \mathrm{P}=0.000)$ and $\mathrm{HHCC}(t=22.47, \mathrm{P}$ $=0.008) P T E N$-overexpression groups were significantly increased. 

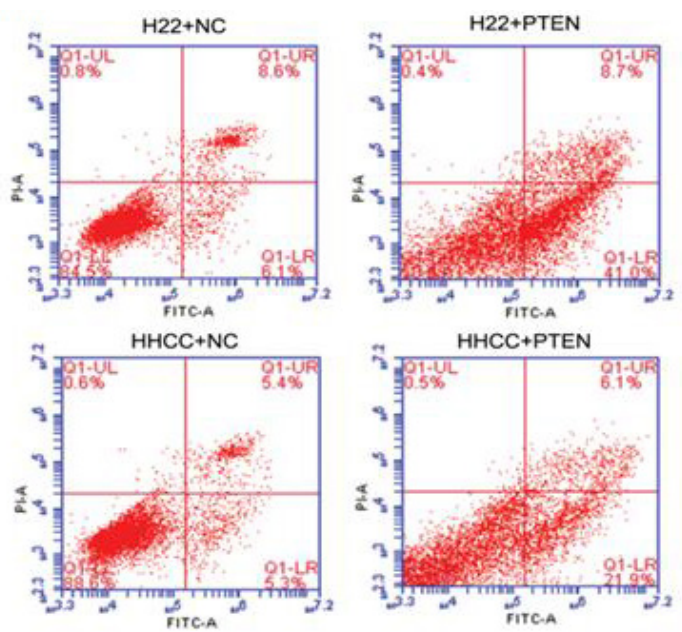

Figure 4. Cell apoptosis measured by flow cytometry. PI = propidium iodide; FITC = fluorescein isothiocyanate.

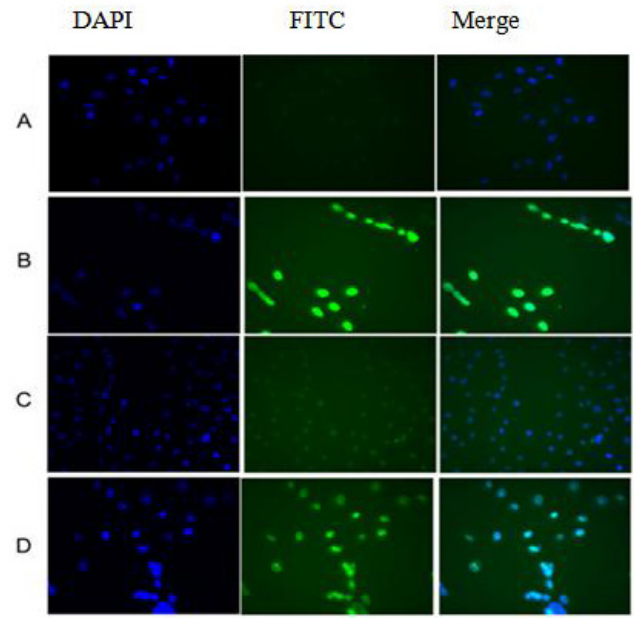

Figure 5. Effect of PTEN overexpression on $\mathrm{H} 22$ and HHCC cell apoptosis, measured by terminal deoxynucleotidyl transferase-mediated deoxyuridine triphosphate nick-end labeling assay. A. H22 control group, B. H22 PTEN overexpression group, C. HHCC control group, D. HHCC PTEN overexpression group. DAPI = 4',6-diamidino-2phenylindole; FITC = fluorescein isothiocyanate.

\section{DISCUSSION}

PTEN can induce apoptosis, inhibit invasion and metastasis of tumor cells and tumor angiogenesis, and maintain immune system stability, among other functions (Zhao et al., 2013). It has been shown that the tumor suppressive effect of $P T E N$ is principally achieved through regulation of the PI3K/AKT signaling pathway and inhibition of those involving FRAP/mTOR, FAK/P130Cas, and APK (Ohta et al., 2015). The PTEN gene has been reported to regulate the proliferation and apoptosis of liver cancer cells, and can maintain normal cellular physiology, 
including metabolism, proliferation, differentiation, and apoptosis by inhibiting AKT activity in the PI3K/AKT signaling pathway (Yamamoto et al., 2013). As a result of PTEN mutation or deletion, PI3K signal transduction may be enhanced, and AKT excessively activated, resulting in hepatocyte overproliferation or even vicious transformation (Yan et al., 2015). Furthermore, PTEN can reduce the phosphorylation of FAK, regulate P130Cas levels, and inhibit cell adhesion and metastasis. As a result, this gene may prevent tumor migration and diffusion (Ohta et al., 2015). Microvessel density is significantly higher in cancer tissue with inactive PTEN than in that with normal PTEN. Moreover, it has been demonstrated that the mutation or inactivation of this gene can enhance expression of endothelial growth factors (including VEGF) and matrix metalloproteinases, and augment the formation of tumor vasculature (He et al., 2016). Liu et al. (2012) explored the effect of docetaxel and a mannan-modified adenovirus encoding PTEN on tumor growth in a murine model, finding that such combined treatment may constitute an effective potential adjuvant therapy for hepatocellular carcinoma.

In this study, we constructed a eukaryotic expression plasmid containing the PTEN gene. The sequence of this construct was found to correspond exactly to that given in GenBank, confirming its successful assembly. Our experiment included four groups, based on two different expression vectors (empty or carrying PTEN) and two cell types (H22 and HHCC). Our results showed that $P T E N$ mRNA was strongly expressed in cells treated with pcDNA3.1PTEN, demonstrating successful transfection of both HHCC and H22 cells. In addition, PTEN protein levels were high in HHCC and H22 cells transfected with pcDNA3.1-PTEN, and significantly so compared to the control groups consisting of cells expressing the pcDNA3.1 empty vector. The apoptosis rate of HHCC cells transfected with the pcDNA3.1-PTEN construct was approximately $21.9 \%$, while that of $\mathrm{H} 22$ cells treated with the same plasmid was around $41.0 \%$. These values were significantly higher than those of the corresponding control groups transfected with the empty vector.

In conclusion, transfection can be successfully used to import a recombinant plasmid containing the PTEN gene into HHCC and H22 cells. Overexpression of PTEN can inhibit proliferation of these cells and effectively promote their apoptosis. Therefore, it is possible to use PTEN transfection to provide new insights into the treatment of liver cancer.

\section{Conflicts of interest}

The authors declare no conflict of interest.

\section{ACKNOWLEDGMENTS}

Research supported by grants from the Department of Science and Technology of Sichuan Province, China (\#SZ0022 to D.D. Zhang), and the Sichuan Academy of Medical Science and Sichuan Provincial People's Hospital (\#30305030611 to M.F. Li).

\section{REFERENCES}

Atreya CE, Sangale Z, Xu N, Matli MR, et al. (2013). PTEN expression is consistent in colorectal cancer primaries and metastases and associates with patient survival. Cancer Med. 2: 496-506. http://dx.doi.org/10.1002/cam4.97

Carletti M, Montani M, Meschini V, Bianchi M, et al. (2015). Stochastic modelling of PTEN regulation in brain tumors: A model for glioblastoma multiforme. Math. Biosci. Eng. 12: 965-981. http://dx.doi.org/10.3934/mbe.2015.12.965 
Chen Y, van de Vijver MJ, Hibshoosh H, Parsons R, et al. (2016). PTEN and NEDD4 in Human Breast Carcinoma. Pathol. Oncol. Res. 22: 41-47. http://dx.doi.org/10.1007/s12253-015-9971-2

Clements AE, Bravo V, Koivisto C, Cohn DE, et al. (2015). WWP2 and its association with PTEN in endometrial cancer. Gynecol Oncol Rep 13: 26-29. http://dx.doi.org/10.1016/j.gore.2015.05.004

Dragoj M, Milosevic Z, Bankovic J, Dinic J, et al. (2015). Association of CCND1 overexpression with KRAS and PTEN alterations in specific subtypes of non-small cell lung carcinoma and its influence on patients' outcome. Tumour Biol. 36: 8773-8780. http://dx.doi.org/10.1007/s13277-015-3620-y

Gupta GP and Massagué J (2006). Cancer metastasis: building a framework. Cell 127: 679-695. http://dx.doi.org/10.1016/j. cell.2006.11.001

He Z, Chen AY, Rojanasakul Y, Rankin GO, et al. (2016). Gallic acid, a phenolic compound, exerts anti-angiogenic effects via the PTEN/AKT/HIF-1 $\alpha$ /VEGF signaling pathway in ovarian cancer cells. Oncol. Rep. 35: 291-297.

Liu Z, Li J, Li J, Huang J, et al. (2012). Mannan-modified Ad5-PTEN treatment combined with docetaxel improves the therapeutic effect in $\mathrm{H} 22$ tumor-bearing mice. Int. J. Nanomedicine 7: 5039-5049.

Manda KR, Tripathi P, Hsi AC, Ning J, et al. (2015). NFATc1 promotes prostate tumorigenesis and overcomes PTEN lossinduced senescence. Oncogene http://dx.doi.org/10.1038/onc.2015.389.

Ohta K, Hoshino H, Wang J, Ono S, et al. (2015). MicroRNA-93 activates c-Met/PI3K/Akt pathway activity in hepatocellular carcinoma by directly inhibiting PTEN and CDKN1A. Oncotarget 6: 3211-3224. http://dx.doi. org/10.18632/oncotarget.3085

Sendor AB, Hacker KE, Chen S, Corona AL, et al. (2015). Von Hippel-Lindau status influences phenotype of liver cancers arising from PTEN loss. Gastrointest Cancer 5: 61-71.

Shearn CT and Petersen DR (2015). Understanding the tumor suppressor PTEN in chronic alcoholism and hepatocellular carcinoma. Adv. Exp. Med. Biol. 815: 173-184. http://dx.doi.org/10.1007/978-3-319-09614-8 10

Siegel RL, Miller KD and Jemal A (2015). Cancer statistics, 2015. CA Cancer J. Clin. 65: 5-29. http://dx.doi.org/10.3322/ caac. 21254

Thomas MB, Jaffe D, Choti MM, Belghiti J, et al. (2010). Hepatocellular carcinoma: consensus recommendations of the National Cancer Institute Clinical Trials Planning Meeting. J. Clin. Oncol. 28: 3994-4005. http://dx.doi.org/10.1200/ JCO.2010.28.7805

Wang DY, Liu L, Qi XS, Su CP, et al. (2015). Hepatic re-resection versus transarterial chemoembolization for the treatment of recurrent hepatocellular carcinoma after initial resection: a systematic review and meta-analysis. Asian Pac. J. Cancer Prev. 16: 5573-5578. http://dx.doi.org/10.7314/APJCP.2015.16.13.5573

Xi Y and Chen Y (2015). Oncogenic and Therapeutic Targeting of PTEN Loss in Bone Malignancies. J. Cell. Biochem. 116: 1837-1847. http://dx.doi.org/10.1002/jcb.25159

Yamamoto R, Shimamoto K, Ishii Y, Kimura M, et al. (2013). Involvement of PTEN/Akt signaling and oxidative stress on indole-3-carbinol (I3C)-induced hepatocarcinogenesis in rats. Exp. Toxicol. Pathol. 65: 845-852. http://dx.doi. org/10.1016/j.etp.2012.12.003

Yamazaki K, Eng C, Kuznetsov SA, Reinisch J, et al. (2015). Missense mutation in the PTEN promoter of a patient with hemifacial hyperplasia. Bonekey Rep. 4: 654. http://dx.doi.org/10.1038/bonekey.2015.21

Yan SY, Chen MM, Li GM, Wang YQ, et al. (2015). MiR-32 induces cell proliferation, migration, and invasion in hepatocellular carcinoma by targeting PTEN. Tumour Biol. 36: 4747-4755. http://dx.doi.org/10.1007/s13277-015$\underline{3124-9}$

Zhao B, Zhu Y, Cui K, Gao J, et al. (2013). Expression and significance of PTEN and miR-92 in hepatocellular carcinoma. Mol. Med. Rep. 7: 1413-1416. 